\title{
Demonstration of a dual-pass differential Fabry-Perot interferometer for future interferometric space gravitational wave antennas
}

\author{
Koji Nagano ${ }^{1,2}$, Hiroki Takeda ${ }^{3}$, Yuta Michimura ${ }^{3}$, Takashi \\ Uchiyama $^{4}$ \& Masaki Ando ${ }^{3,5}$ \\ ${ }^{1}$ KAGRA Observatory, Institute for Cosmic Ray Research, The University of Tokyo, \\ Kashiwa, Chiba 277-8582, Japan \\ ${ }^{2}$ Institute of Space and Astronautical Science, Japan Aerospace Exploration Agency, \\ Sagamihara, Kanagawa 252-5210, Japan \\ ${ }^{3}$ Department of Physics, Graduate School of Science, The University of Tokyo, \\ Bunkyo, Tokyo 113-0033, Japan \\ ${ }^{4}$ KAGRA Observatory, Institute for Cosmic Ray Research, The University of Tokyo, \\ Hida, Gifu 506-1205, Japan \\ ${ }^{5}$ Research Center for the Early Universe, The University of Tokyo, Bunkyo, Tokyo \\ 113-0033, Japan \\ E-mail: knagano@ac.jaxa.jp
}

August 2020

\begin{abstract}
A dual-pass differential Fabry-Perot interferometer (DPDFPI) is one candidate of the interferometer configurations utilized in future Fabry-Perot type space gravitational wave antennas, such as Deci-hertz Interferometer Gravitational Wave Observatory. In this paper, the working principle of the DPDFPI has been investigated and necessity to adjust the absolute length of the cavity for the operation of the DPDFPI has been found. In addition, using the 55-cm-long prototype, the operation of the DPDFPI has been demonstrated for the first time and it has been confirmed that the adjustment of the absolute arm length reduces the cavity detuning as expected. This work provides the proof of concept of the DPDFPI for application to the future Fabry-Perot type space gravitational wave antennas.
\end{abstract}

\section{Introduction}

The first detection of the gravitational wave from the black hole binary by the Advanced Laser Interferometer Gravitational-Wave Observatory (aLIGO) opened the era of the gravitational wave physics and astronomy [1]. The first detection has been followed by many detections of the gravitational wave from the black hole and neutron star binaries [2, 3]. The gravitational wave detections and its electromagnetic followup observations have already provided significant physical and astronomical information [4, 5]. 
For further expansion of the gravitational wave physics and astronomy, we need to observe manifold classes of the gravitational wave objects. In other words, expanding the observation frequency is required [6, 7, 8]. Although stellar mass objects, $\sim 1-100$ $M_{\odot}\left(M_{\odot}\right.$ is the solar mass), have been observed by aLIGO around $100 \mathrm{~Hz}$, a relatively heavy object, e.g. $\sim 10^{3} M_{\odot}$ is still attractive observational target around $0.1 \mathrm{~Hz}[9,10]$.

The upper observable mass bound, i.e. lower limit of the observation frequency range, of aLIGO and the other ground based detector is mainly limited by ground motion [11, 12. Thus, in order to observe the heavy objects, space gravitational wave antennas have been proposed, such as Laser Interferometer Space Antenna [13], Big Bang Observer [14], TianQin [15], Taiji [16], TianGO [17], and DECi-hertz Interferometer Gravitational Wave Observatory (DECIGO) [18].

Among them, DECIGO and its precursor proposal, B-DECIGO [19], are planning to utilize a Fabry-Perot interferometer to enhance the sensitivity around $0.1 \mathrm{~Hz}$. The Fabry-Perot interferometer gives DECIGO the possibility even to observe a stochastic gravitational wave background generated in the early Universe [20, 21, 22, 23]. Therefore, when the Fabry-Perot type space gravitational wave observatory is realized, new gravitational wave physical and astronomical knowledge will be provided by the observation of the astronomical objects that has not been detected.

There are, however, some challenges for use of the Fabry-Perot cavity in space mission. One of the challenges is how to ensure the redundancy with as a small number of components as possible. In space missions, the total mass of the components is critical since it is strongly restricted by the ability of the launch system. One proposed solution to ensure the redundancy is a dual-pass Fabry-Perot interferometer configuration [18]. In the configuration, the Fabry-Perot cavity is designed to be critically coupled and two lasers from two sources are injected to the cavity from both cavity mirrors. Consequently, the cavity signal can be measured with both lasers. As a result, the redundancy of the interferometer operation is provided by the minimum number of the mirrors.

For further proceedings of the dual-pass Fabry-Perot interferometer concept in the gravitational wave detector, it was necessary to investigate a realistic proposal on the concept. In this paper, we propose a realistic dual-pass FabryPerot interferometer interferometer configuration, a dual-pass differential FabryPerot Interferometer (DPDFPI). Figure 1 shows the schematic of the DPDFPI. In the DPDFPI, the gravitational wave signal is mainly obtained with a differential Fabry-Perot interferometer, which is adopted in some ground-based gravitational wave detectors [24, 25]. As another configuration with the dual-pass Fabry-Perot interferometer, a back-linked Fabry-Perot interferometer was also proposed [26]. It uses two laser sources in one satellite for each cavity metrology and the gravitational wave signal is obtained by making two lasers interfere in one satellite. Compared with the back-linked Fabry-Perot interferometer, the DPDFPI requires the relatively simple optical configuration without the back-link interferometer. However, in the DPDFPI shown in fig. 1, we need to consider a new control topology among three cavities peculiar 


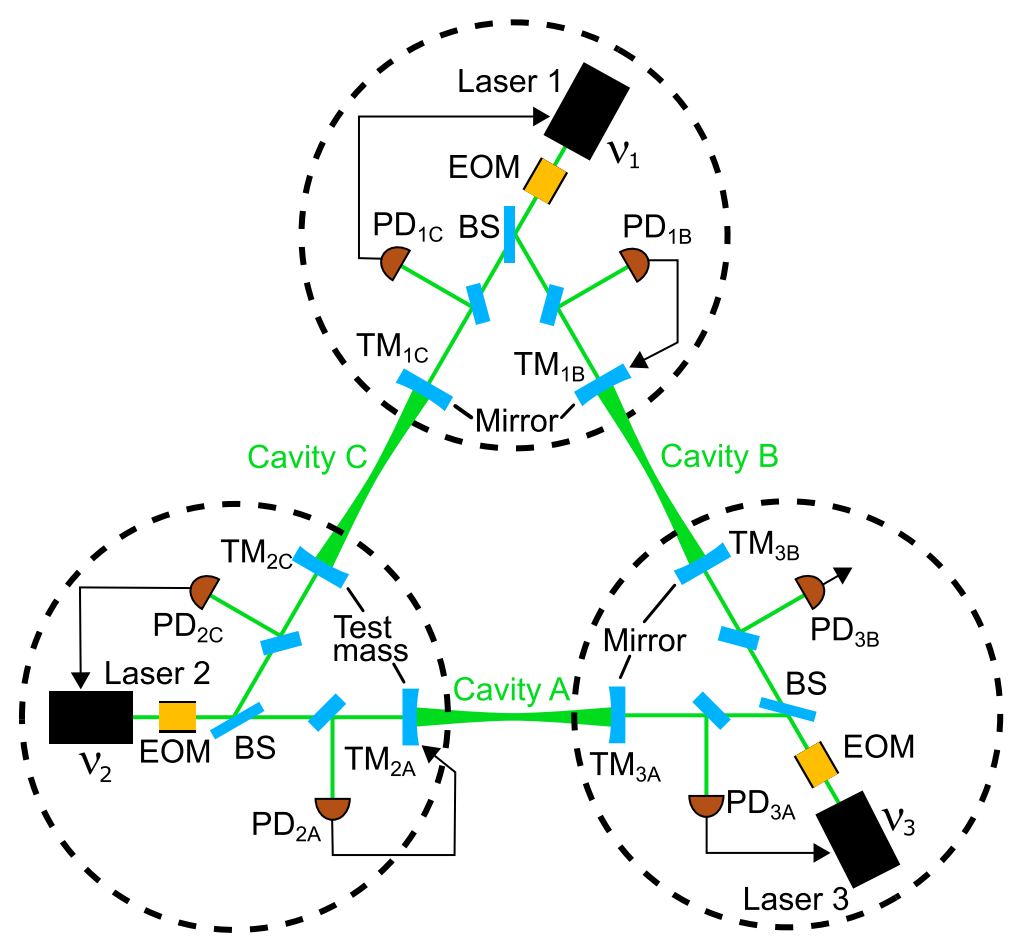

Figure 1. Schematic view of the DPDFPI considered for DECIGO. PD is a photodetector, EOM is an electro-optic modulator, BS is a beam splitter, and TM is a test mass. Instruments in the dashed circle are placed in one station, i.e. one satellite in the space detector. For decoupling, the frequency of the lasers are shifted for each other and the polarization of the lasers input to one cavity is orthogonalised.

to the DPDFPI since the cavity mirrors are shared with each other interferometer. In this paper, we analytically investigate the working principle of the DPDFPI for the first time and show the requirement of the cavity length adjustment for the operation of the DPDFPI. Moreover, we constructed the first experimental prototype of the DPDFPI for its proof of concept.

\section{Formalization of the dual-pass differential Fabry-Perot interferometer}

We present the working principle of the DPDFPI using the block diagram. Figure 2 shows the block diagram of the DPDFPI shown in fig. 1. For the measurement with the Fabry-Perot cavity, we need to make it resonate using, for example, Pound-Drever-Hall technique [27]. Usually, servo system is used to keep the resonance. Notice that the frequency of the lasers in the DPDFPI are shifted for each other for decoupling. The signal flow of the DPDFPI shown in fig. 1 is presented in table 1. The length signal, i.e. the resonant frequency, of the Cavity $\mathrm{C}$ is fed back to the Laser 1 and 2. With this feed back system, the frequency of the Laser 1 and 2 are controlled at the resonant frequency of the Cavity C. Then, the resonant frequency of the Cavity A and B are compared with the frequency of the Laser 1 and 2, respectively, and are controlled by actuating the position of the mirrors, $\mathrm{TM}_{2 \mathrm{~A}}$ and $\mathrm{TM}_{1 \mathrm{~B}}$, respectively. In addition, the 
Table 1. Signal flow of the DPDFPI shown in fig. 11 The signal obtained with the photodetectors in the first column corresponds to the sensing objects in the second column and is fed back to the controller in the third column.

\begin{tabular}{lll}
\hline Photodetector & Sensing object & Controller \\
\hline $\mathrm{PD}_{1 \mathrm{~B}}$ & Laser 1, Cavity B & $\mathrm{TM}_{1 \mathrm{~B}}$ \\
$\mathrm{PD}_{1 \mathrm{C}}$ & Laser 1, Cavity C & Laser 1 \\
$\mathrm{PD}_{2 \mathrm{C}}$ & Laser 2, Cavity C & Laser 2 \\
$\mathrm{PD}_{2 \mathrm{~A}}$ & Laser 2, Cavity A & $\mathrm{TM}_{2 \mathrm{~A}}$ \\
$\mathrm{PD}_{3 \mathrm{~A}}$ & Laser 3, Cavity A & Laser 3 \\
$\mathrm{PD}_{3 \mathrm{~B}}$ & Laser 3, Cavity B & - \\
\hline
\end{tabular}

length signal of the Cavity A is fed back to the Laser 3 with the consequence that the frequency of the Laser 3 is controlled at the resonant frequency of the Cavity A. Thanks to the above feedback system, the Laser 1 (2) resonates with the Cavity B and C (A and $\mathrm{C}$ ) and the Laser 3 resonates with the Cavity A. One consideration is that the length signal measured with the $\mathrm{PD}_{3 \mathrm{~A}}$ cannot be fed back to the length of the Cavity $\mathrm{B}$ or the frequency of the Laser 2 since the feedback paths are occupied by the other signals. Therefore, we need some method to make the Laser 3 resonate with the Cavity B as discussed later. If the Laser 3 resonates with the Cavity B, the obtained signals in the feedback system shown in fig. 2 are expressed as,

$$
\begin{aligned}
s_{\mathrm{PD}_{1 \mathrm{~B}}}= & \frac{1}{1+G_{1 \mathrm{~B}}}\left(-\frac{G_{\nu_{1}}}{1+G_{\nu_{1}}} \frac{L_{\mathrm{B}}}{L_{\mathrm{C}}} \Delta x_{\mathrm{C}}+\Delta x_{\mathrm{B}}+\frac{1}{1+G_{\nu_{1}}} L_{\mathrm{B}} \frac{\delta \nu_{1}}{\nu_{1}}\right), \\
s_{\mathrm{PD}_{1 \mathrm{C}}}= & \frac{1}{1+G_{\nu_{1}}}\left(\Delta x_{\mathrm{C}}+L_{\mathrm{C}} \frac{\delta \nu_{1}}{\nu_{1}}\right), \\
s_{\mathrm{PD}_{2 \mathrm{C}}}= & \frac{1}{1+G_{\nu_{2}}}\left(\Delta x_{\mathrm{C}}+L_{\mathrm{C}} \frac{\delta \nu_{2}}{\nu_{2}}\right), \\
s_{\mathrm{PD}_{2 \mathrm{~A}}}= & \frac{1}{1+G_{2 \mathrm{~A}}}\left(-\frac{G_{\nu_{2}}}{1+G_{\nu_{2}}} \frac{L_{\mathrm{A}}}{L_{\mathrm{C}}} \Delta x_{\mathrm{C}}+\Delta x_{\mathrm{A}}+\frac{1}{1+G_{\nu_{2}}} L_{\mathrm{A}} \frac{\delta \nu_{2}}{\nu_{2}}\right), \\
s_{\mathrm{PD}_{3 \mathrm{~A}}}= & \frac{1}{1+G_{\nu_{3}}}\left(\frac{G_{\nu_{2}}}{1+G_{\nu_{2}}} \frac{G_{2 \mathrm{~A}}}{1+G_{2 \mathrm{~A}}} \frac{L_{\mathrm{A}}}{L_{\mathrm{C}}} \Delta x_{\mathrm{C}}+\frac{1}{1+G_{2 \mathrm{~A}}} \Delta x_{\mathrm{A}}\right. \\
& \left.\quad+L_{\mathrm{A}} \frac{\delta \nu_{3}}{\nu_{3}}-\frac{1}{1+G_{\nu_{2}}} \frac{G_{2 \mathrm{~A}}}{1+G_{2 \mathrm{~A}}} L_{\mathrm{A}} \frac{\delta \nu_{2}}{\nu_{2}}\right), \\
s_{\mathrm{PD}_{3 \mathrm{~B}}}= & \left(-\frac{G_{\nu_{2}}}{1+G_{\nu_{2}}} \frac{G_{2 \mathrm{~A}}}{1+G_{2 \mathrm{~A}}} \frac{G_{\nu_{3}}}{1+G_{\nu_{3}}}+\frac{G_{\nu_{2}}}{1+G_{\nu_{2}}} \frac{G_{1 \mathrm{~B}}}{1+G_{1 \mathrm{~B}}}\right) \frac{L_{\mathrm{B}}}{L_{\mathrm{C}}} \Delta x_{\mathrm{C}} \\
& -\frac{1}{1+G_{2 \mathrm{~A}}} \frac{G_{\nu_{3}}}{1+G_{\nu_{3}}} \frac{L_{\mathrm{B}}}{L_{\mathrm{A}}} \Delta x_{\mathrm{A}}+\frac{1}{1+G_{1 \mathrm{~B}}} \Delta x_{\mathrm{B}}+\frac{1}{1+G_{\nu_{3}}} L_{\mathrm{B}} \frac{\delta \nu_{3}}{\nu_{3}} \\
& -\frac{1}{1+G_{\nu_{1}}} \frac{G_{1 \mathrm{~B}}}{1+G_{1 \mathrm{~B}}} L_{\mathrm{B}} \frac{\delta \nu_{1}}{\nu_{1}}+\frac{1}{1+G_{\nu_{2}}} \frac{G_{2 \mathrm{~A}}}{1+G_{2 \mathrm{~A}}} \frac{G_{\nu_{3}}}{1+G_{\nu_{3}}} L_{\mathrm{B}} \frac{\delta \nu_{2}}{\nu_{2}},
\end{aligned}
$$


where $\Delta x_{\mathrm{A}} \equiv x_{3 \mathrm{~A}}-x_{2 \mathrm{~A}}, \Delta x_{\mathrm{B}} \equiv x_{1 \mathrm{~B}}-x_{3 \mathrm{~B}}$, and $\Delta x_{\mathrm{C}} \equiv x_{2 \mathrm{C}}-x_{1 \mathrm{C}}$ are the cavity length fluctuation, $x_{i \alpha}(i=1,2,3$ and $\alpha=\mathrm{A}, \mathrm{B}, \mathrm{C})$ are the longitudinal displacement of each test mass, $\nu_{i}$ are the laser frequency of the Laser $i, \delta \nu_{i}$ are its fluctuation, $L_{\alpha}$ are the cavity length of the Cavity $\alpha, s_{\mathrm{PD}_{i \alpha}}$ are the signal obtained with each photodetector, and $G$ are the open loop gain. When $\left|G_{\nu_{i}}\right| \gg 1$ and $\left|G_{i \alpha}\right| \ll 1$ (or $G_{2 \mathrm{~A}} \simeq G_{1 \mathrm{~B}}$ ), $s_{\mathrm{PD}_{2 \mathrm{~A}}}$, $s_{\mathrm{PD}_{1 \mathrm{~B}}}$, and $s_{\mathrm{PD}_{3 \mathrm{~B}}}$, are denoted as

$$
\begin{aligned}
& s_{\mathrm{PD}_{1 \mathrm{~B}}}=\frac{1}{1+G_{1 \mathrm{~B}}}\left(-\Delta x_{\mathrm{C}}+\Delta x_{\mathrm{B}}\right), \\
& s_{\mathrm{PD}_{2 \mathrm{~A}}}=\frac{1}{1+G_{2 \mathrm{~A}}}\left(-\Delta x_{\mathrm{C}}+\Delta x_{\mathrm{A}}\right), \\
& s_{\mathrm{PD}_{3 \mathrm{~B}}}= \begin{cases}-\Delta x_{\mathrm{A}}+\Delta x_{\mathrm{B}} & \left(\left|G_{1 \mathrm{~B}}\right| \ll 1,\left|G_{2 \mathrm{~A}}\right| \ll 1\right) \\
\frac{1}{1+G_{1 \mathrm{~B}}}\left(-\Delta x_{\mathrm{A}}+\Delta x_{\mathrm{B}}\right) & \left(G_{2 \mathrm{~A}} \simeq G_{1 \mathrm{~B}}\right)\end{cases}
\end{aligned}
$$

Here, we assume that all arm cavities have almost the same length, L. Equations (7)-(9) indicate that differential signals between two cavities, which include gravitational wave signals, can be obtained in the DPDFPI.

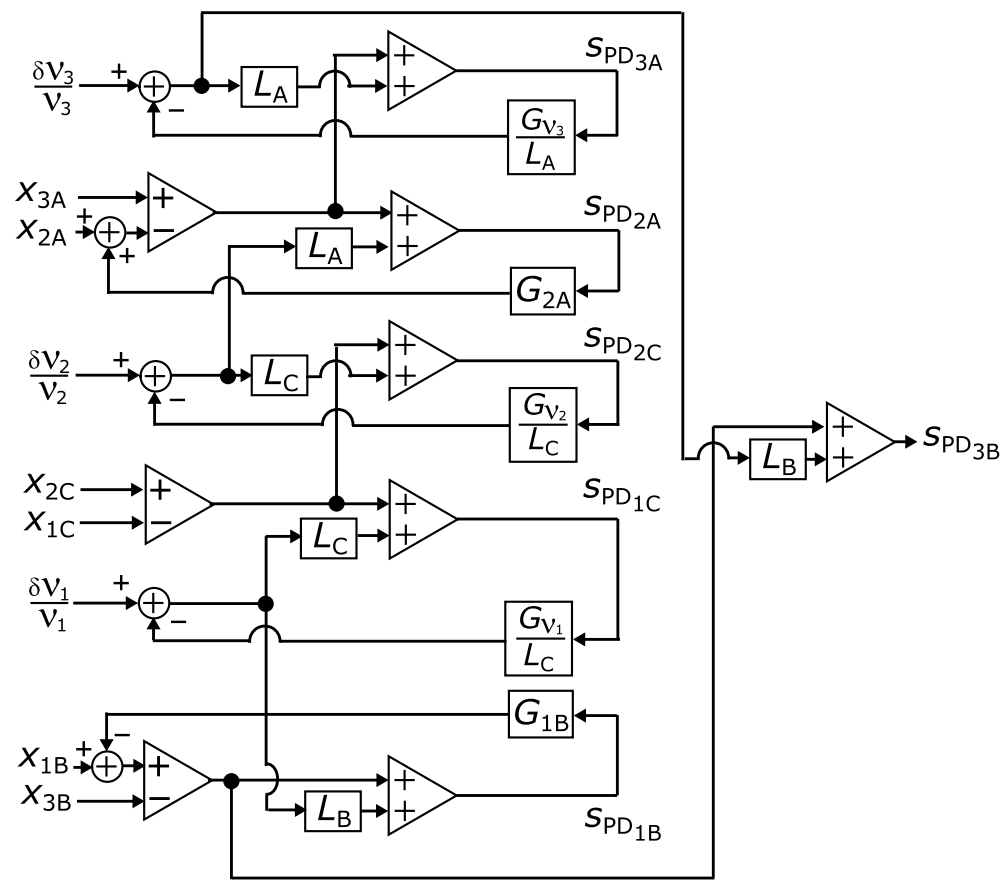

Figure 2. Block diagram of the DPDFPI shown in fig. 1 $x_{i \alpha}(i=1,2,3$ and $\alpha=\mathrm{A}, \mathrm{B}, \mathrm{C})$ are the longitudinal displacement of each test mass, $\nu_{i}$ are the laser frequency of the Laser $i, \delta \nu_{i}$ are its fluctuation, $L_{\alpha}$ are the cavity length of the Cavity $\alpha, s_{\mathrm{PD}_{i \alpha}}$ are the signal obtained with each photodetector, and $G$ are the open loop gain.

Here, we explain how to make the Laser 3 resonate with the Cavity B. Let us consider the frequency offset of the Laser $2, \Delta \nu_{3}$, from the resonant frequency of the 
Cavity B, $N \frac{c}{2 L_{\mathrm{B}}}(N \in \mathbb{N}) . \Delta \nu_{3}$ is expressed by

$$
\Delta \nu_{3} \equiv \nu_{3}-N \frac{c}{2 L_{\mathrm{B}}},
$$

where $L_{\mathrm{B}}$ is the length of the Cavity $\mathrm{B}$ and $c$ is the speed of light. Since the frequency of the Laser 3 is controlled to follow the resonant frequency of the Cavity $\mathrm{A}, \nu_{3}$ is written as

$$
\nu_{3}=N^{\prime} \frac{c}{2 L_{\mathrm{A}}} \quad\left(N^{\prime} \in \mathbb{N}\right),
$$

where $L_{\mathrm{A}}$ is the length of the Cavity A. Hence, $\Delta \nu_{3}$ is denoted as

$$
\Delta \nu_{3}=N^{\prime} \frac{c}{2 L_{\mathrm{A}}}-N \frac{c}{2 L_{\mathrm{B}}} .
$$

When the length of the Cavity $\mathrm{A}$ is $L_{\mathrm{A}}=L_{\mathrm{B}}+\Delta L\left(|\Delta L| \ll L_{\mathrm{B}}\right), \Delta \nu_{3}$ is written as

$$
\Delta \nu_{3}=\frac{c}{2 L_{\mathrm{B}}}\left(\Delta N-N \frac{\Delta L}{L_{\mathrm{B}}}\right),
$$

where $\Delta N \equiv N^{\prime}-N$. By choosing $\Delta N$ to be the proper integer to $-N \frac{\Delta L}{L_{\mathrm{B}}}$ by adjusting $\nu_{3}, \Delta \nu_{3}$ is constrained to be within

$$
\left|\Delta \nu_{3}\right| \leq \frac{c|\Delta L|}{2 L_{\mathrm{B}}^{2}} .
$$

This is because, when we change $\Delta N \rightarrow \Delta N+1$ and $N \rightarrow N+1, \Delta \nu_{3}$ is changed by the difference of the free spectral range of the Cavity $\mathrm{A}$ and $\mathrm{B}$ as

$$
\left|\frac{c}{2 L_{\mathrm{A}}}-\frac{c}{2 L_{\mathrm{B}}}\right|=\frac{c|\Delta L|}{2 L_{\mathrm{B}}^{2}} .
$$

Here, we use the fact of $|\Delta L| \ll L_{\mathrm{B}}$.

In order to resonate the Cavity B with the Laser $3, \Delta \nu_{3}$ has to be well within the linewidth of the Cavity B. Consequently, eq. (14) indicates that we need to adjust $\Delta L$ for the resonance. For example, in DECIGO and B-DECIGO, the cavity linewidth is $15 \mathrm{~Hz}$ [19]. Thus we need to adjust the cavity length to be $\Delta L \ll 100 \mathrm{~km}$ for DECIGO $(L=1000 \mathrm{~km})$ and $\Delta L \ll 1 \mathrm{~km}$ for B-DECIGO $(L=100 \mathrm{~km})$ for the operation of the DPDFPI. Moreover, for the reduction of the interferometer noise coupled with the detuning, e.g. laser intensity coupling noise, the requirement for $\Delta L$ can be strict depending on the sensitivity requirement. For example, if the laser intensity coupling noise is considered, $\Delta L<\frac{2 L_{\mathrm{B}}^{2} h_{\mathrm{req}}}{c I_{\mathrm{RIN}}} \nu_{3}$ where $h_{\text {req }}$ is the sensitivity requirement, and $I_{\mathrm{RIN}}$ is a relative intensity noise of the input laser.

\section{Experimental setup for the demonstration of the DPDFPI}

Since the DPDFPI is the new interferometer configuration, the experimental demonstration of the DPDFPI is necessary. Especially, the following two points 


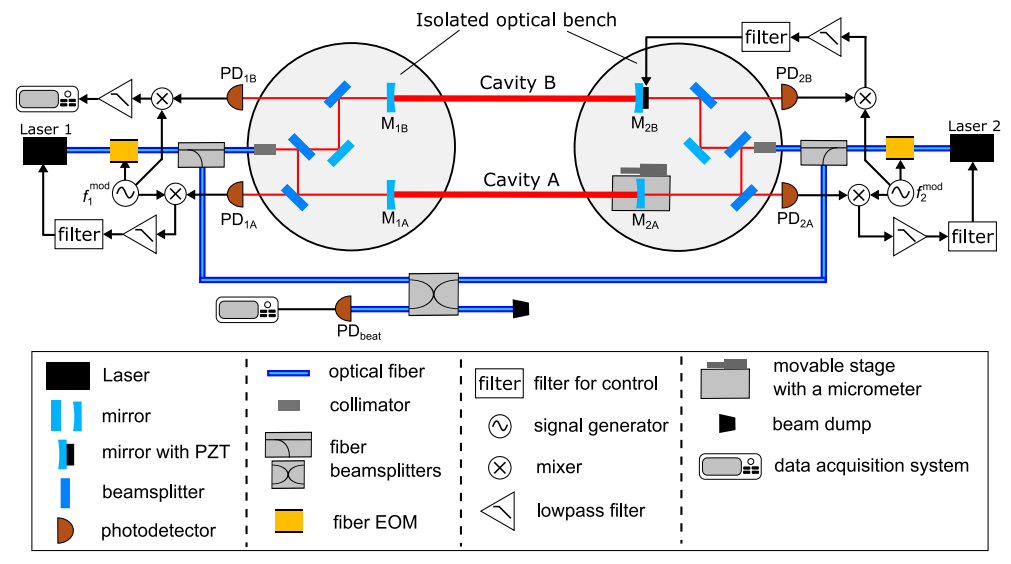

Figure 3. Schematic of the DPDFPI experiment.

should be confirmed: first, dependence of the laser frequency offset from the cavity resonant frequency on the cavity length difference discussed in the previous section, and, second, the operation of the DPDFPI, i.e. the measurement of the differential cavity displacement signal. For the experimental demonstration, we construct the prototype of the DPDFPI. Figure 3 shows the schematic of the experiment of the DPDFPI prototype. In the DPDFPI prototype, only two cavities are used since the operation of the DPDFPI can be confirmed by evaluating the correlation of the signals measured with two lasers. Even in this setup, the key feature of the DPDFPI, i.e. the necessity of the cavity length adjustment for the interferometer operation, still remains. Thus, we need to adjust the length of the Cavity A against the length of the Cavity B to operate the interferometer.

In the experiment, we use two laser sources, Koheras AdjustiK C15 (Laser 1) and Koheras BASIK X15 (Laser 2), with a wavelength of $1550 \mathrm{~nm}$. The output power of the Laser 1 and the Laser 2 are $10 \mathrm{~mW}$ and $30 \mathrm{~mW}$, respectively. The laser beams are phase modulated with electro-optic modulators for the Pound-Drever-Hall technique [27. After the electro-optic modulators, the laser beams are splitted into two ways. One beam is injected to the main interferometer, i.e. the DPDFPI, and another beam is injected to the auxiliary interferometer for the cavity absolute length measurement, which is explained in Appendix A. The length of the two cavities is measured to be $0.55340 \pm 0.00001 \mathrm{~m}$. The length of the Cavity $\mathrm{A}$ is able to be adjusted using the stage with the movable stage. For the main cavities, the lasers are injected from both sides. The main cavities are composed of the mirrors having the same specification. Their radius of curvature is $2 \mathrm{~m}$ and their amplitude reflectivity is 0.992 . The reflected and transmitted beams from the cavities are measured with the photodetectors and the cavity longitudinal signals are obtained with the Pound-Drever-Hall technique [27]. The two laser frequencies are shifted by one free spectral range of the Cavity $\mathrm{A}, \frac{c}{2 L_{\mathrm{A}}}$. The cavity mirrors are placed on the optical bench that is isolated with the rubber stack. The resonant frequency of the optical bench is about $10 \mathrm{~Hz}$. 


\section{Results and discussions of the demonstration experiment}

We investigated the dependence of the frequency difference, $\Delta \nu$, between the frequency of the Laser 1 and the resonant frequency of the Cavity B on the cavity length difference between the Cavity A and B. $\Delta \nu$ corresponds to $\Delta \nu_{3}$ in the previous discussion in Section 2. The measured result is shown in fig. 4 . Figure 4 shows that $\Delta \nu$ is shifted depending on the cavity length difference. From fig. 4, the relation between the frequency offset and the cavity length difference is determined to be $(-5.1 \pm 0.5) \times 10^{8} \mathrm{~Hz} / \mathrm{m}$ by linear fitting the measured data. The determined value is consistent with the expected value, $(-4.8976 \pm 0.0002) \times 10^{8} \mathrm{~Hz} / \mathrm{m}$, from eq. (15) and the measured cavity length within the error ranges.

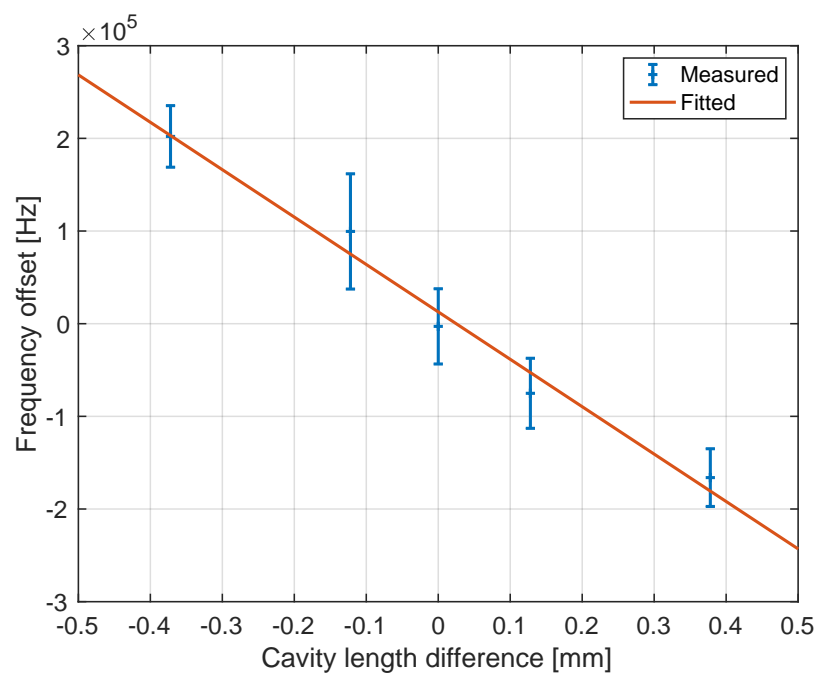

Figure 4. Measured frequency difference, $\Delta \nu$, between the frequency of the Laser 1 and the resonant frequency of the Cavity B. The solid line is determined by fitting the measured data.

After the cavity length adjustment, the noise spectra of the interferometer were measured as shown in figure 5. The ' $1 \mathrm{~B}$ ' and ' $2 \mathrm{~B}$ ' curves represent the spectra measured with $\mathrm{PD}_{1 \mathrm{~B}}$ and $\mathrm{PD}_{2 \mathrm{~B}}$, respectively. In the proper condition of the open loop gain, the spectra shown in fig. 5 indicate the differential length fluctuations of the Cavity A and the Cavity B as discussed in Section 2. Note that the unity gain frequency of the frequency control loop of the Laser 1 and the Laser 2 were measured to be $2.9 \mathrm{kHz}$ and $4.0 \mathrm{kHz}$, respectively.

If the DPDFPI in fig. 3 is properly operated, the correlated differential signals between the Cavity $\mathrm{A}$ and the Cavity $\mathrm{B}$ are measured with the $\mathrm{PD}_{1 \mathrm{~B}}$ and the $\mathrm{PD}_{2 \mathrm{~B}}$. To check the correlation, the coherence between the signals from the $\mathrm{PD}_{1 \mathrm{~B}}$ and the $\mathrm{PD}_{2 \mathrm{~B}}$ is calculated as shown in fig. 6. Given the average number of 100 for the calculation, the $95 \%$ significance threshold of the coherence is 0.06 [28. Thus, fig. 6 indicates that the two signals are coherent below $\sim 1.5 \mathrm{kHz}$ as expected from the unity gain frequency 


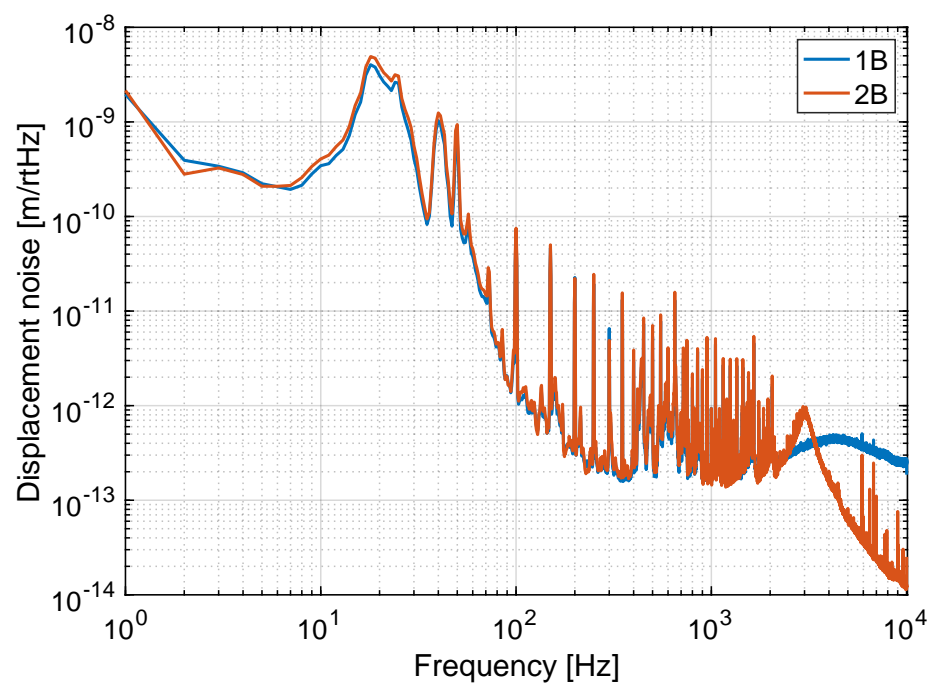

Figure 5. Calibrated noise spectra of the DPDFPI measured with the $\mathrm{PD}_{1 \mathrm{~B}}$ and the $\mathrm{PD}_{2 \mathrm{~B}}$.

of the frequency control loops of the Laser 1 and the Laser 2. Hence, the DPDFPI is conceived properly operated.

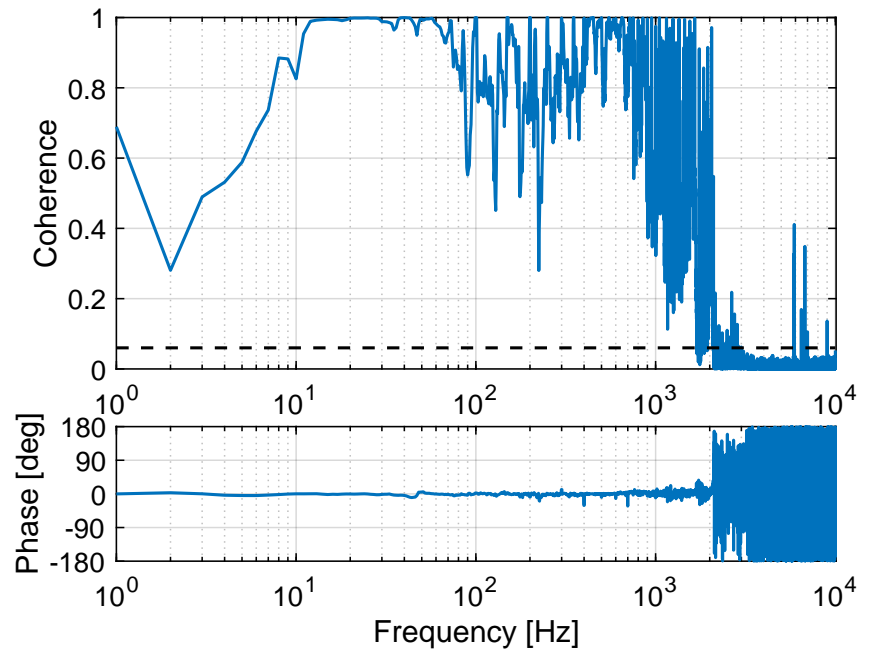

Figure 6. Measured magnitude-squared amplitude (upper panel) and phase (lower panel) of the coherence between the signals from the $\mathrm{PD}_{1 \mathrm{~B}}$ and the $\mathrm{PD}_{2 \mathrm{~B}}$. The dashed line is the significance threshold of the coherence, $0.06[28]$.

\section{Conclusion}

In this paper, we presented the working principle of the DPDFPI for the first time. For the operation of the DPDFPI, the absolute length adjustment is necessary. Moreover, 
using the 55-cm-long DPDFPI prototype, we demonstrated the operation of the DPDFPI and confirmed that adjustment of the absolute arm length reduced the cavity detuning as expected with our formulation. This work provides the proof of concept of the DPDFPI for application to the future Fabry-Perot type space gravitational wave antennas.

\section{Acknowledgements}

We thank Kiwamu Izumi, Tomofumi Shimoda, Yutaro Enomoto, and Ayaka Shoda for fruitful discussions. We appreciate the technical support from Shigemi Otsuka, and Togo Shimozawa in the machine shop of Graduate School of Science, The University of Tokyo. We also thank Kunihiko Hasegawa and Takaharu Shishido for preparing some mechanical components in the experiment. This work is financially supported by JSPS Grant-in-Aid for Scientific Research (A) No. 15 H02087 and JSPS KAKENHI Grant No. JP17J01176.

\section{Appendix A. Cavity absolute length measurement}

Here, we explain how to measure the absolute length of the cavity in our experiment. We adopt a similar scheme to [29, 30]. In the dual-pass cavity, two different lasers resonate with a cavity. Thus, by measuring the frequency difference between the two lasers, we are able to determine the cavity free spectral range, which is related with the cavity length. For example, in our experimental setup shown in fig. 3, the frequency difference of the Laser 1 and the Laser $2, \nu_{\text {diff }}$, is expressed as

$$
\nu_{\text {diff }}=n \frac{c}{2 L_{\mathrm{A}}}=n^{\prime} \frac{c}{2 L_{\mathrm{B}}}\left(n, n^{\prime} \in \mathbb{N}\right) .
$$

Note that $\frac{c}{2 L_{\mathrm{A}}}$ and $\frac{c}{2 L_{\mathrm{B}}}$ are the free spectral range of the Cavity $\mathrm{A}$ and the Cavity $\mathrm{B}$, respectively. When we know $n\left(n^{\prime}\right)$, the cavity length $L_{\mathrm{A}}\left(L_{\mathrm{B}}\right)$ can be determined by measuring $\nu_{\text {diff }} . \nu_{\text {diff }}$ is measured by observing the interference between the two lasers. In this work, $n$ and $n^{\prime}$ are set to be 1 and the interference signal between the Laser 1 and the Laser 2 is measured with the $\mathrm{PD}_{\text {beat }}$ in fig. 3 .

It is worth noting that, even in the setup shown in fig. 1, the cavity length can be measured with almost the same scheme used in fig. 3. One difference is using the injected and transmitted laser of the cavity to obtain the interference signal. Although two injected lasers are interfered in this work, it is challenging to do the same thing in the space detectors where two laser sources are placed in the distant satellites.

\section{References}

[1] B. P. Abbott et al., "Observation of Gravitational Waves from a Binary Black Hole Merger", Physical Review Letters 116, 061102 (2016). 
[2] B. P. Abbott et al., "GWTC-1: A Gravitational-Wave Transient Catalog of Compact Binary Mergers Observed by LIGO and Virgo during the First and Second Observing Runs", Physical Review X 9, 031040 (2019).

[3] B. P. Abbott et al., "GW170817: Observation of Gravitational Waves from a Binary Neutron Star Inspiral", Physical Review Letters 119, 161101 (2017).

[4] B. P. Abbott et al., "Gravitational Waves and Gamma-Rays from a Binary Neutron Star Merger: GW170817 and GRB 170817A", The Astrophysical Journal 848, L13 (2017).

[5] B. P. Abbott et al., "Multi-messenger Observations of a Binary Neutron Star Merger", The Astrophysical Journal 848, L12 (2017).

[6] A. Sesana, "Prospects for Multiband Gravitational-Wave Astronomy after GW150914", Physical Review Letters 116, 231102 (2016).

[7] S. Vitale, "Multiband Gravitational-Wave Astronomy: Parameter Estimation and Tests of General Relativity with Space- and Ground-Based Detectors", Physical Review Letters 117, 051102 (2016).

[8] S. Isoyama, H. Nakano, and T. Nakamura, "Multiband gravitational-wave astronomy: Observing binary inspirals with a decihertz detector, B-DECIGO", Progress of Theoretical and Experimental Physics 2018 (2018).

[9] T. Matsubayashi, H. Shinkai, and T. Ebisuzaki, "Gravitational Waves from Merging IntermediateMass Black Holes", The Astrophysical Journal 614, 864 (2004).

[10] C. Reisswig et al., "Formation and Coalescence of Cosmological Supermassive-Black-Hole Binaries in Supermassive-Star Collapse", Physical Review Letters 111, 151101 (2013).

[11] S. A. Hughes and K. S. Thorne, "Seismic gravity-gradient noise in interferometric gravitationalwave detectors", Physical Review D 58, 122002 (1998).

[12] R. Abbott et al., "Seismic isolation enhancements for initial and advanced LIGO", Classical and Quantum Gravity 21, S915 (2004).

[13] P. Amaro-Seoane et al., "Laser Interferometer Space Antenna", arXiv:1702.00786 [astro-ph] (2017).

[14] G. M. Harry, P. Fritschel, D. A. Shaddock, W. Folkner, and E. S. Phinney, "Laser interferometry for the Big Bang Observer", Classical and Quantum Gravity 23, 4887 (2006).

[15] J. Luo et al., "TianQin: a space-borne gravitational wave detector", Classical and Quantum Gravity 33, 035010 (2016).

[16] W.-R. Hu and Y.-L. Wu, "The Taiji Program in Space for gravitational wave physics and the nature of gravity", National Science Review 4, 685 (2017).

[17] K. A. Kuns, H. Yu, Y. Chen, and R. X. Adhikari, "Astrophysics and cosmology with a deci-hertz gravitational-wave detector: TianGO", arXiv:1908.06004 [astro-ph, physics:gr-qc] (2019).

[18] S. Kawamura et al., "The Japanese space gravitational wave antenna: DECIGO", Classical and Quantum Gravity 28, 094011 (2011).

[19] S. Kawamura et al., "Current status of space gravitational wave antenna DECIGO and BDECIGO", arXiv:2006.13545 [gr-qc] (2020).

[20] T. L. Smith, M. Kamionkowski, and A. Cooray, "Direct detection of the inflationary gravitationalwave background", Physical Review D 73, 023504 (2006).

[21] J. García-Bellido, D. G. Figueroa, and A. Sastre, "Gravitational wave background from reheating after hybrid inflation", Physical Review D 77, 043517 (2008).

[22] S. Kuroyanagi, K. Nakayama, and S. Saito, "Prospects for determination of thermal history after inflation with future gravitational wave detectors", Physical Review D 84, 123513 (2011).

[23] L. Alabidi, K. Kohri, M. Sasaki, and Y. Sendouda, "Observable spectra of induced gravitational waves from inflation", Journal of Cosmology and Astroparticle Physics 2012, 017 (2012).

[24] A. Abramovici et al., "Improved sensitivity in a gravitational wave interferometer and implications for LIGO", Physics Letters A 218, 157 (1996).

[25] M. Ohashi et al., "Design and construction status of CLIO", Classical and Quantum Gravity 20, S599 (2003). 
[26] K. Izumi and M. Fujimoto, "A back-linked Fabry-Perot interferometer for space-borne gravitational wave observations", Submitted to The Astrophysical Journal (2020).

[27] R. W. P. Drever et al., "Laser phase and frequency stabilization using an optical resonator", Applied Physics B 31, 97 (1983).

[28] R. O. R. Y. Thompson, "Coherence Significance Levels", Journal of Atmospheric Sciences 36, 2020 (1979).

[29] A. Araya et al., "Absolute-length determination of a long-baseline Fabry-Perot cavity by means of resonating modulation sidebands", Applied Optics 38, 2848 (1999).

[30] A. Stochino, K. Arai, and R. X. Adhikari, "Technique for in situ measurement of free spectral range and transverse mode spacing of optical cavities", Applied Optics 51, 6571 (2012). 